\title{
Sensor Pose Estimation from Multi-center Cylindrical Panoramas
}

\author{
Fay Huang $^{1}$, Reinhard Klette ${ }^{2}$, and Yun-Hao Xie ${ }^{1}$ \\ ${ }^{1}$ Institute of Computer Science and Information Engineering, \\ National Ilan University, Taiwan, R.O.C. \\ fay@niu.edu.tw \\ ${ }^{2}$ Department of Computer Science, \\ The University of Auckland, New Zealand \\ r.klette@auckland.ac.nz
}

\begin{abstract}
Cylindrical panoramas can be classified into various types according to their basic scanning properties and mutual spatial alignment, such as single-center (e.g., as in QTVR), concentric, multi-center, symmetric, or (after a transformation onto a cylinder) catadioptric panoramas. This paper deals with a solution of the sensor pose estimation problem using (somehow calculated) corresponding points in the multi-center panoramas. All other types of panoramas are able to be described by this general multi-center model. Due to the non-linearity of the multi-centered projection geometry, the modeling of sensor pose estimation typically results into non-linear and highly complicated forms which incur numerical instability. This paper shows that there exist linear models for sensor pose estimation under minor geometrical constraints, namely symmetric and leveled panoramas. The presented approaches are important for solving the 3D data fusion problem for multiple panoramas; it is also fundamental for an in-depth analysis of multi-view geometry of panoramic images.
\end{abstract}

\section{Introduction}

Panoramic images have been studied with respect to $3 \mathrm{D}$ scene visualization, navigation and reconstruction for more than a decade. Applications include stereoscopic visualization, stereo reconstruction, walkthrough or virtual reality.

Various types of panoramic images are proposed to match particular applications. This paper focuses on those using a cylindrical representation. See Table 1 for a classification of cylindrical panoramas and their applications. A $360^{\circ}$ cylindrical panoramic image can be acquired by various means, such as a rotating video or matrix-sensor camera, a catadioptric sensor (with a subsequent mapping onto a cylinder), or a rotating sensor-line camera, as commercially available from various producers since the late 1990s. For simplifying our discussion, we assume a model close to the latter one which has a fixed rotation axis and takes images consecutively at equidistant angles. (Rotating sensor-line cameras allow maximum accuracy, and have been used, e.g., in major architectural photogrammetric projects; see [1]). The projection center of the camera does not have to 
Table 1. Classification of cylindrical panoramas

\begin{tabular}{|l|l|l|}
\hline (A) Multi-view Panoramas & $\begin{array}{l}\text { Most general case, it is for } \\
\text { geometrical studies of finite } \\
\text { sets of cylindric panoramas. }\end{array}$ \\
\hline $\begin{array}{l}\text { (B) Parallel-axis Panoramas } \\
\text { (e.g. Leveled Panoramas) }\end{array}$ & $\begin{array}{l}\text { Virtual Reality Tour } \\
\text { Exhibitions } \\
\text { Surveillance System }\end{array}$ \\
\hline (C) Co-axis Panoramas & & $\begin{array}{l}\text { 3D Scene Reconstruction } \\
\text { Virtual Reality (a high ceiling }\end{array}$ \\
\hline (E) Symmentront)
\end{tabular}

be on the rotation axis. The major advantage of such off-axis distance is to allow acquiring stereo panoramas that is stereo viewable. The resulting panoramic images in this case, while the off-axis distance is greater than zero, are refereed to as multi-center panoramas.

A multi-view panorama [2] is a set of multi- or single-center cylindrical panoramas which were recorded at different locations and/or with different capturing parameters. In particular, they might be acquired with respect to different rotation axes. In comparison to a single axial panorama 34567, the advantages of multi-view panoramas are known to include enlarged visibility and improved stereo reconstruction opportunities; in short, they define multi-view image analysis for the cylindrical panoramic case.

A panoramic image is recorded by a panoramic sensor. Sensor pose estimation deals with recovering the relative pose of two (calibrated) sensors. Compared to planar images or catadioptric images, there is very few literature on sensor pose estimation from cylindrical panoramas.

Ishikuro el at. 8] dealt with a very restricted case of the sensor pose estimation problem, in which the given panoramas are acquired at the same altitude and with parallel rotation axes. Kang and Szeliski [9] discussed the sensor pose estimation problem only for single-center panoramas. Neither generalized multiview cases (i.e., different intrinsic sensor parameter values and arbitrary sensor poses) nor practically relevant cases (e.g., the multi-view panoramas in [57]) of sensor pose estimation have been studied or discussed in the literatures before. This paper provides (for the first time) a cost function whose minimization solves the pose estimation problem for two standard cases of multi-view (and thus also multi-center) cylindrical panoramas.

In the following sections, we first briefly review the chosen basic sensor model and notions used in the paper. Sensor pose estimation from a general multi-view 


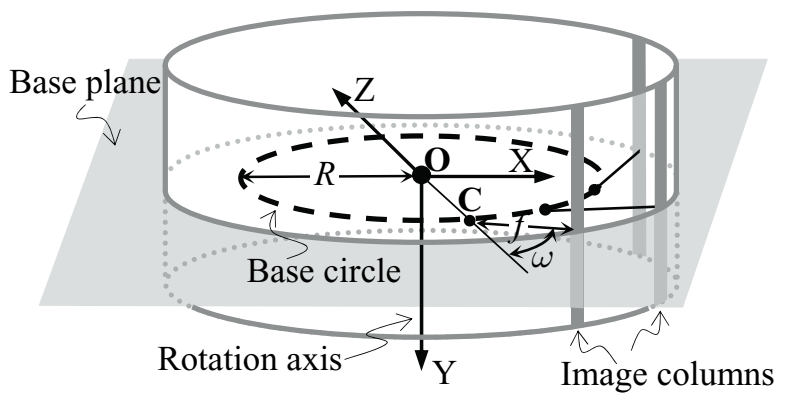

Fig. 1. Basic sensor model of multi-center cylindrical panoramas: the origin of the sensor coordinate system is at $\mathbf{O}$. Three image columns are shown with their projection centers.

cylindrical panorama is discussed in Section 3. Two general cases of multi-view panoramas are elaborated in Section 4. Section 5 informs about experiments using recorded or synthetic images. There are also concluding remarks.

\subsection{Sensor Model and Notations}

The sensor model used generalizes various panoramic imaging models 348. The model consists of multiple projection centers and a cylindrical image surface; see Fig. 1. $\mathbf{C}$ denotes a projection center. Projection centers are uniformly distributed on the base circle. This circle is incident with the base plane. $\mathbf{O}$ denotes the center of the base circle; it is also the origin of the sensor coordinate system. The off-axis distance $R$ (radius of the base circle) describes the distance between any projection center and the rotation axis.

A cylindrical panorama is partitioned into image columns of equal width which are parallel to the rotation axis. The number of image columns is the width $W$ of the panorama. There is a one-to-one ordered mapping between image columns and projection centers. The distance between a projection center and its associated image column is called the effective focal length, and is denoted by $f$ (see Fig. 1). The principal angle $\omega$ is between a projection ray in the base plane, emitting from $\mathbf{C}$, and the normal vector of the base circle at point $\mathbf{C}$. The four intrinsic sensor parameters, $R, f, \omega$, and $W$ characterize how a panoramic image $E_{\mathcal{P}}(R, f, \omega, W)$ is acquired.

Consider two panoramas, $E_{\mathcal{P}_{1}}$ and $E_{\mathcal{P}_{2}}$. The geometric relationship between both sensor coordinate systems can be described by a $3 \times 3$ rotation matrix $\mathbf{R}$ and a $3 \times 1$ translation vector $\mathbf{T}$. The rotation matrix is given by three row vectors $\left[\mathbf{r}_{1}^{\mathrm{T}} \mathbf{r}_{2}^{\mathrm{T}} \mathbf{r}_{3}^{\mathrm{T}}\right]^{\mathrm{T}}$, and the translation vector equals $\left(t_{x}, t_{y}, t_{z}\right)^{\mathrm{T}}$.

\section{General Multi-view Case}

Consider a pair of panoramas, $E_{\mathcal{P}_{1}}\left(R_{1}, f_{1}, \omega_{1}, W_{1}\right)$ and $E_{\mathcal{P}_{2}}\left(R_{2}, f_{2}, \omega_{2}, W_{2}\right)$, which are taken at arbitrary poses in $3 \mathrm{D}$ space. Let $\left(x_{1}, y_{1}\right)$ and $\left(x_{2}, y_{2}\right)$ denote 


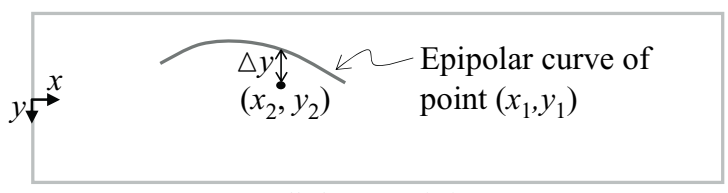

Unrolled panoramic image

Fig. 2. Row difference $\Delta y$ between the actual corresponding image point $\left(x_{2}, y_{2}\right)$ and the point where epipolar curve and column $x_{2}$ intersect

the image coordinates of the projection of a 3D point in these two panoramas, respectively. If multiple pairs of corresponding image points are provided, say $\left(x_{1 i}, y_{1 i}\right)$ and $\left(x_{2 i}, y_{2 i}\right)$, for $i=1,2, \ldots, n$, then we are able to estimate sensor poses by minimizing the following cost function,

$$
\min \sum_{i=1}^{n}\left(y_{2 i}-\frac{f_{2} \mathbf{r}_{2}^{\mathrm{T}} \cdot \mathbf{V}}{\sin \delta_{2 i} \mathbf{r}_{1}^{\mathrm{T}} \cdot \mathbf{V}+\cos \delta_{2 i} \mathbf{r}_{3}^{\mathrm{T}} \cdot \mathbf{V}-R_{2} \cos \omega_{2}}\right)^{2}
$$

where $\alpha_{k i}=\frac{2 \pi x_{k i}}{W_{k}}, \delta_{k i}=\left(\alpha_{k i}+\omega_{k}\right), \beta_{k i}=\tan ^{-1}\left(\frac{y_{k i}}{f_{k}}\right)$, and $k=1$ or 2. Moreover,

$$
\begin{aligned}
& \mathbf{V}=\mathbf{A}+\frac{R_{2} \sin \omega_{2}+\cos \delta_{2 i} \mathbf{r}_{1}^{\mathrm{T}} \cdot \mathbf{A}-\sin \delta_{2 i} \mathbf{r}_{3}^{\mathrm{T}} \cdot \mathbf{A}}{\sin \delta_{2 i} \mathbf{r}_{3}^{\mathrm{T}} \cdot \mathbf{B}-\cos \delta_{2 i} \mathbf{r}_{1}^{\mathrm{T}} \cdot \mathbf{B}} \mathbf{B} \\
& \mathbf{A}=\left(\begin{array}{c}
R_{1} \sin \alpha_{1 i}-t_{x} \\
-t_{y} \\
R_{1} \cos \alpha_{1 i}-t_{z}
\end{array}\right) \text { and } \mathbf{B}=\left(\begin{array}{c}
\sin \delta_{1 i} \cos \beta_{1 i} \\
\sin \beta_{1 i} \\
\cos \delta_{1 i} \cos \beta_{1 i}
\end{array}\right)
\end{aligned}
$$

The cost function is defined to be the image row difference $\triangle y$, each between the actual corresponding image point in column $x$, and the point where epipolar curve and column $x$ intersect. See Fig. 2 for an unrolled panoramic image. Epipolar curves are calculated based on point coordinates $x_{1 i}, y_{1 i}$ and sensor parameters; see [2]. Note that in our assumed sensor model, the epipolar curve can be considered to be a function $y=G(x)$, which is either monotonically increasing (decreasing), has exactly one turning point $(\max / \mathrm{min})$, or is always equals to a constant.

In this general case, the three row vectors of the rotation matrix and the three elements of the translation vector are "distributed" within the given complex cost function. The estimation of sensor poses appears to be rather difficult, if not impossible for the unrestricted case.

\section{Two Standard Multi-view Cases}

However, when using panoramic sensors, such as rotating sensor-line systems, then it is actually standard to aim for a set of leveled panoramas, and for symmetric panoramas if stereo-viewing is also intended; see [1. (The latter case also simplifies stereo reconstruction.) 


\subsection{Two Symmetric Pairs}

$E_{\mathcal{P}_{1}}(R, f, \omega, W)$ and $E_{\mathcal{P}_{2}}(R, f,-\omega, W)$ define a symmetric pair of panoramas, both defined on the same sensor coordinate system. (Symmetric pairs can easily be acquired using a single off-shelf camera, e.g., see the approach in [5].) Epipolar curves are in this case lines which may be identified with image rows (see proofs in 27[10]). Therefore, dense image correspondences can be calculated by using stereo matching algorithms as developed for stereo pairs of planar images.

If $3 \mathrm{D}$ data are collected from multiple pairs of symmetric panoramas, acquired at different locations, then data fusion becomes a challenge, and the registration step requires that the sensor pose estimation problem to be solved in advance.

The basic idea of our sensor pose estimation approach is as follows: first, for each symmetric pair, transform pairs of corresponding image points into directional unit vectors pointing to the reconstructed 3D points; second, establish a geometric relation between these two bunches of unit vectors that are respectively defined in two sensor coordinate systems. The following theorem shows how the directional unit vector of a $3 \mathrm{D}$ point (with respect to $\mathbf{O}$ ) is derived from a pair of corresponding points $\left(x_{1}, y\right)$ and $\left(x_{2}, y\right)$ on symmetric panoramas $E_{\mathcal{P}_{1}}(R, f, \omega, W)$ and $E_{\mathcal{P}_{2}}(R, f,-\omega, W)$. Let $\mathbf{u}$ be the directional unit vector of that $3 \mathrm{D}$ point which projects into $\left(x_{1}, y\right)$ and $\left(x_{2}, y\right)$.

Theorem 1. This directional vector can be calculated as follows:

$$
\boldsymbol{u}=\frac{\left(\sin \omega \sin \alpha, \frac{y}{f} \sin \beta, \sin \omega \cos \alpha\right)^{T}}{\sqrt{\sin ^{2} \omega+\frac{y^{2}}{f^{2}} \sin ^{2} \beta}}
$$

where $\alpha=\frac{\left(x_{1}+x_{2}\right) \pi}{W}$ and $\beta=\frac{\left(x_{2}-x_{1}\right) \pi}{W}$.

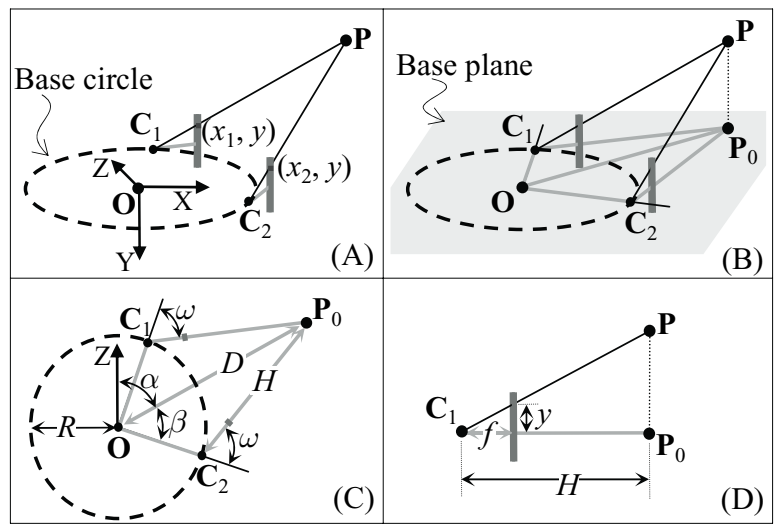

Fig. 3. Geometric interpretation of directional unit vector calculation, as described in the proof of Theorem 1 


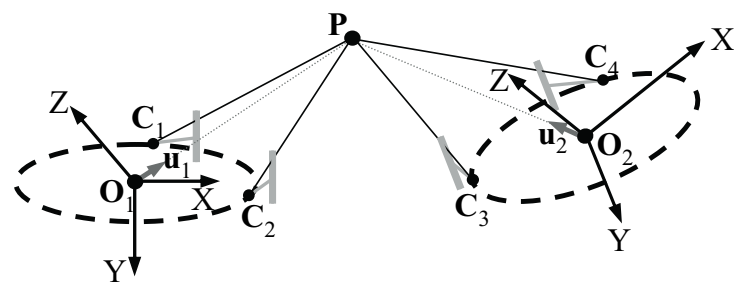

Fig. 4. Four corresponding points in two pairs of symmetric panoramas and its preimage $\mathbf{P}$ in $3 \mathrm{D}$ space. Vectors $\mathbf{u}_{1}$ and $\mathbf{u}_{2}$ are two corresponding directional unit vectors of $\mathbf{P}$ with respect to the sensor coordinate systems $\mathbf{O}_{1}$ and $\mathbf{O}_{2}$, respectively.

Proof. Let $\mathbf{P}=\left(P_{X}, P_{Y}, P_{Z}\right)$ be a $3 \mathrm{D}$ point that projects into image points $\left(x_{1}, y\right)$ and $\left(x_{2}, y\right)$ on panoramas $E_{\mathcal{P}_{1}}$ and $E_{\mathcal{P}_{2}}$, respectively, see Fig. 3(A). Let $\mathbf{P}_{0}$ denote the projection of point $\mathbf{P}$ on the base plane, as illustrated in Fig. 3(B). Define $\alpha$ as the angle between the $Z$-axis and line segment $\overline{\mathbf{O P}} \bar{P}_{0}$, and $\beta$ be the angle of $\angle \mathbf{P}_{0} \mathbf{O} \mathbf{C}_{1}$ (or $\left.\angle \mathbf{P}_{0} \mathbf{O} \mathbf{C}_{2}\right)$, see Fig. $3(\mathrm{C})$. Then we have that $\alpha=\frac{\left(x_{1}+x_{2}\right) \pi}{W}$ and $\beta=\frac{\left(x_{2}-x_{1}\right) \pi}{W}$. Moreover, define $H$ to be the length of the line segment $\overline{\mathbf{P}_{0} \mathbf{C}_{1}}$ (or $\overline{\mathbf{P}_{0} \mathbf{C}_{2}}$ ), and $D$ to be the length of the line segment $\overline{\mathbf{O P}_{0}}$. We have that $D=\frac{R \sin \omega}{\sin (\omega-\beta)}$. Hence, $P_{X}=\frac{R \sin \omega \sin \alpha}{\sin (\omega-\beta)}$ and $P_{Z}=\frac{R \sin \omega \cos \alpha}{\sin (\omega-\beta)}$. Furthermore, we have that $H=\frac{R \sin \beta}{\sin (\omega-\beta)}$, and we know the property $\frac{H}{f}=\frac{\overline{\mathbf{P P}_{0}}}{y}$ (see Fig. 3(D)), where $\overline{\mathbf{P P}_{0}}=P_{Y}$. It follows that $P_{Y}=\frac{y R \sin \beta}{f \sin (\omega-\beta)}$. After a normalization of vector $\left(P_{X}, P_{Y}, P_{Z}\right)$, the directional unit vector $\mathbf{u}$ of $3 \mathrm{D}$ point $\mathbf{P}$ follows as given in the theorem.

Theorem 1 can directly be applied to approaches in [2] and [5] where angle $\omega$ can take any value. However, for the approach in [7, where the direction of projection is tangential to the base circle (i.e., $\omega=90^{\circ}$ ), the formula in Theorem 1 can even be simplified and is given in the following corollary.

Corollary 1. If $\omega=\frac{\pi}{2}$, then we have that

$$
\boldsymbol{u}=\frac{\left(\sin \alpha, \frac{y}{f} \sin \beta, \cos \alpha\right)^{T}}{\sqrt{1+\frac{y^{2}}{f^{2}} \sin ^{2} \beta}}
$$

where $\alpha=\frac{\left(x_{1}+x_{2}\right) \pi}{W}$ and $\beta=\frac{\left(x_{2}-x_{1}\right) \pi}{W}$.

Note that we do not have any dependence of the off-axis distance $R$ in those two formulas, which dramatically simplifies the estimation of sensor poses. For the sensor pose estimation from two arbitrary symmetric pairs (see Fig. 4), we may apply reasoning and results as in [11].

Theorem 2. Given are at least eight pairs of corresponding points in two pairs of symmetric panoramas, where the associated sensor parameters are known except for $R$. The relative sensor poses can then be recovered by the normalized 8-point algorithm up to a scale factor. 
Proof. Each pair of corresponding image points in a pair of symmetric panoramas can be transformed into an directional unit vector by Theorem 1. Figure 4 shows a point $\mathbf{P}$ that defines two corresponding directional unit vectors $\mathbf{u}_{1}$ and $\mathbf{u}_{2}$ with respect to sensor coordinate systems $\mathbf{O}_{1}$ and $\mathbf{O}_{2}$, respectively.

Assume that coordinate system $\mathbf{O}_{1}$ coincides with the world coordinate system. Let $\mathbf{R}$ and $\mathbf{T}$ describe the orientation and translation of the sensor coordinate system $\mathbf{O}_{2}$ with respect to the world coordinate system. Since any pair of corresponding directional unit vectors is coplanar in 3D (i.e., epipolar constraint), we have that $\mathbf{u}_{1}^{T}\left(\mathbf{T} \times \mathbf{R}^{-1} \mathbf{u}_{2}\right)=0$. We can rewrite this as $\mathbf{u}_{1}^{T}\lfloor\mathbf{T}\rfloor_{\times} \mathbf{R}^{-1} \mathbf{u}_{2}=0$, where $\lfloor\mathbf{T}\rfloor_{\times}$is the skew symmetric matrix of vector $\mathbf{T}$. Then, we have $\mathbf{u}_{1}^{T} \mathbf{E} \mathbf{u}_{2}=0$, where $\mathbf{E}=\lfloor\mathbf{T}\rfloor_{\times} \mathbf{R}^{-1}$. Here, the matrix $\mathbf{E}$ is equivalent to the essential matrix in multiple planar image geometry [11, thus the normalized 8-point algorithm applies to solve the sensor pose estimation problem in this symmetric case.

Hence, we may conclude that eight pairs of corresponding image points are sufficient to determine the relative poses of both sensors up to a scale factor, where the value of sensor parameter $R$ needs not to be known.

Moreover, if all the sensor parameter values (including $R$ ) are pre-calibrated and given, then the exact sensor pose associated to $\mathbf{O}_{2}$ can be recovered with respect to the world coordinate system (defined at $\mathbf{O}_{1}$ ). This is because those parameter values allow to calculate the exact distance between any two 3D points. Then, these distances can be used as a reference to recover the unknown scale factor in Theorem 2 ,

\subsection{One Leveled Pair}

In this second common approach for capturing multi-view panoramas, the only constraint is that all associated base planes have to be parallel (say, to the sea level), to be guaranteed by a lever. Figure 5 sketches a leveled pair of panoramas.

Leveled panoramas are common for virtual navigation [1213 or reconstruction [8,9] of a large scale environments. Leveled panoramas allow large "overlapping" fields of views. The larger the common field of view, the higher the probability that object surfaces are visible in more than one panorama. Hence, this supports more reliable stereo reconstruction and smooth view-transitions between multiple panoramas in a walk-through simulation.

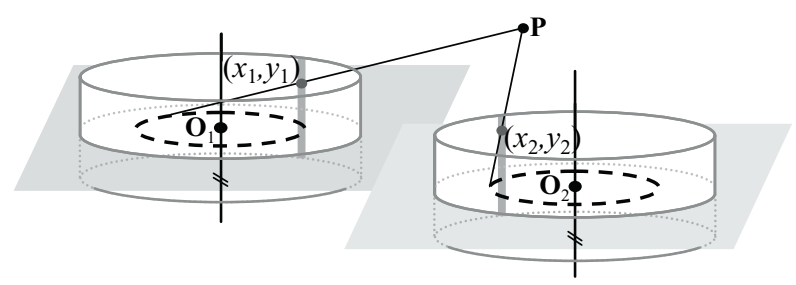

Fig. 5. A pair of leveled panoramas and a pair of corresponding image points 
The sensor pose estimation criteria of a leveled pair is specified in the following theorem. Two leveled panoramas are acquired by sensors with the same intrinsic parameters, and the sensor poses are related by a single rotation angle $\phi$ with respect to the rotation axis and a translation vector $\left(t_{x}, t_{y}, t_{z}\right)^{\mathrm{T}}$. The five variables in this theorem, to be recovered, are $X_{1}=\cos \phi, X_{2}=\sin \phi, X_{3}=t_{x}, X_{4}=t_{z}$, and $X_{5}=t_{y}$. In the equational system we will also use the following nine coefficients:

$$
\begin{aligned}
& c_{1 i}=y_{2 i} R \sin \left(\delta_{1 i}-\alpha_{2 i}\right)+y_{1 i} R \sin \left(\delta_{2 i}-\alpha_{1 i}\right) \\
& c_{2 i}=y_{1 i} R \cos \left(\delta_{2 i}-\alpha_{1 i}\right)-y_{2 i} R \cos \left(\delta_{1 i}-\alpha_{2 i}\right) \\
& c_{3 i}=-y_{2 i} \cos \delta_{1 i} \quad c_{4 i}=y_{2 i} \sin \delta_{1 i} \\
& c_{5 i}=y_{1 i} \cos \delta_{2 i} \quad c_{6 i}=-y_{1 i} \sin \delta_{2 i} \\
& c_{7 i}=f \sin \left(\alpha_{2 i}-\alpha_{1 i}\right) \quad c_{8 i}=f \cos \left(\alpha_{2 i}-\alpha_{1 i}\right) \\
& c_{9 i}=-\left(y_{1 i}+y_{2 i}\right) R \sin \omega
\end{aligned}
$$

where $\alpha_{k i}=\frac{2 \pi x_{k i}}{W}, \delta_{k i}=\left(\alpha_{k i}+\omega\right)$, and $k=1$ or 2 .

Theorem 3. Given a set of corresponding pairs of points $\left(x_{1 i}, y_{1 i}\right)$ and $\left(x_{2 i}, y_{2 i}\right)$, where $i=1,2, \ldots, n$, the values of $\phi, t_{x}, t_{y}$, and $t_{z}$ can be estimated by minimizing the following sum,

$$
\begin{array}{r}
\sum_{i=1}^{n}\left(c_{1 i} X_{1}+c_{2 i} X_{2}+c_{3 i} X_{3}+c_{4 i} X_{4}+c_{5 i} X_{1} X_{3}+c_{6 i} X_{1} X_{4}\right. \\
\left.+c_{7 i} X_{1} X_{5}+c_{6 i} X_{2} X_{3}-c_{5 i} X_{2} X_{4}+c_{8 i} X_{2} X_{5}+c_{9 i}\right)^{2}
\end{array}
$$

subjected to the constraints $X_{1}^{2}+X_{2}^{2}=1, X_{1}^{2} \leq 1$, and $X_{2}^{2} \leq 1$.

Proof. Let $\left(x_{1}, y_{1}\right)$ and $\left(x_{2}, y_{2}\right)$ be a pair of corresponding image points in a pair of leveled cylindrical panoramas $E_{\mathcal{P}_{1}}$ and $E_{\mathcal{P}_{2}}$, respectively. Given $x_{1}$ and $y_{1}$, the corresponding epipolar curve in $E_{\mathcal{P}_{2}}$ can be expressed as follows (see [1]):

$$
\begin{aligned}
& y_{2} R \sin \left(\alpha_{1}+\omega-\alpha_{2}-\phi\right)-y_{2} R \sin \omega-y_{2} \cos \left(\alpha_{1}+\omega\right) t_{x} \\
& +y_{2} \sin \left(\alpha_{1}+\omega\right) t_{z}+f \sin \left(\alpha_{2}-\alpha_{1}+\phi\right) t_{y}-y_{1} R \sin \omega \\
& +y_{1} R \sin \left(\alpha_{2}-\alpha_{1}+\omega+\phi\right)+y_{1} \cos \left(\alpha_{2}+\omega+\phi\right) t_{x} \\
& -y_{1} \sin \left(\alpha_{2}+\omega+\phi\right) t_{z}=0
\end{aligned}
$$

The cost function is defined by the row difference between an actual corresponding image point and the point on the same column incident with the epipolar curve. In short, by algebraic rearrangements of Equation (5), we obtain the second-order algebraic representation for the minimization in this theorem.

\section{Experiments}

Several real-world experiments on estimating sensor poses have been carried out at different places and by using different type of cameras. Camera and sensor 

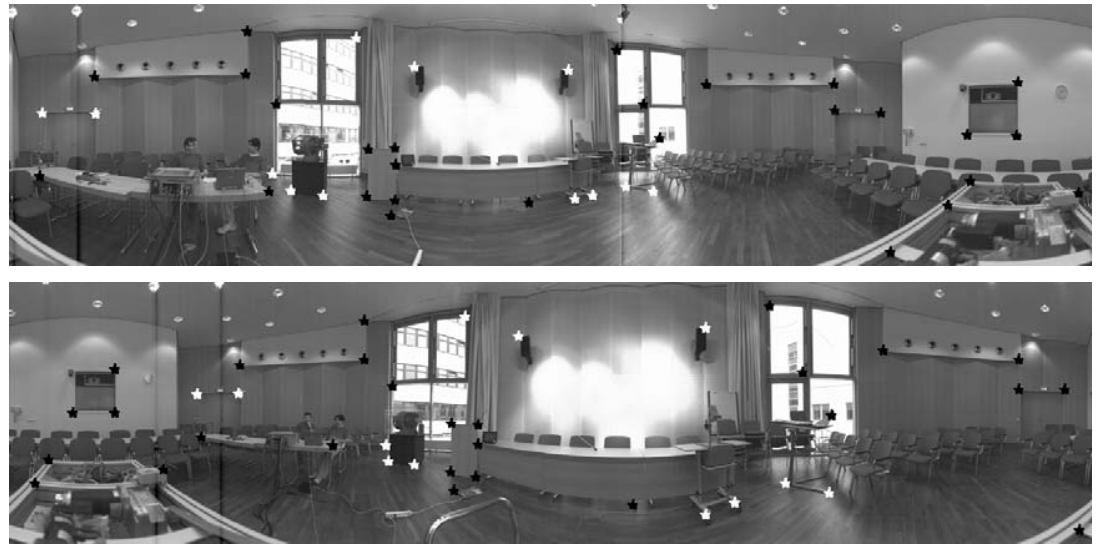

Fig. 6. Two symmetric leveled panorama pairs acquired at different locations (top: right panorama of the first pair, bottom: right panorama of the second pair), all marked with 40 corresponding points
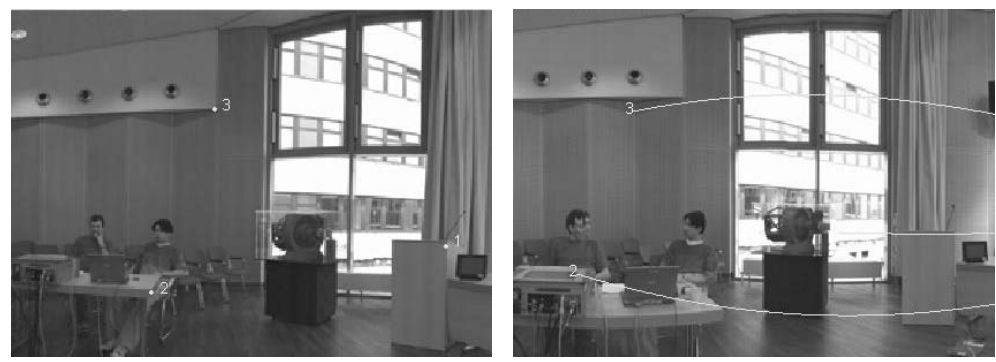

Fig. 7. Illustration of three epipolar curves calculated based on the pose estimation results

were calibrated separately in advance; thus the camera's intrinsic parameters were known and kept unaltered during image acquisition. Figure 6 illustrate one example of a leveled pair taken by a line-camera at different locations in the same room. Actually, at each location, a pair of stereo panoramas were acquired for experiments, and those shown in Fig. 6] are the "right" panoramas only. In this particular example, we used $R=100 \mathrm{~mm}, f=21.7 \mathrm{~mm}$, and $\omega= \pm 155^{\circ}$. Each panorama has an image resolution of $324 \times 1,343$. We identified manually in total 40 corresponding points (marked as stars).

The true rotation matrix $\mathbf{R}$ and translation vector $\mathbf{T}$ of these two symmetric panorama pairs were measured with less than $\pm 1 \%$ error, and we have $\phi=50^{\circ}$ and $\left(t_{x}, t_{y}, t_{z}\right)=(-1,000,-45,-1,000)$ in $\mathrm{mm}$. The estimated sensor pose is denoted as $\hat{\mathbf{R}}$ and $\hat{\mathbf{T}}$. The error measurements for rotation and translation are defined as $\arccos \left(\left(\operatorname{tr}\left(\mathbf{R} \hat{\mathbf{R}}^{\mathrm{T}}\right)-1\right) / 2\right)$ and $\arccos (\mathbf{T} \cdot \hat{\mathbf{T}} /\|\mathbf{T}\|\|\hat{\mathbf{T}}\|)$, respectively, both in degrees. 

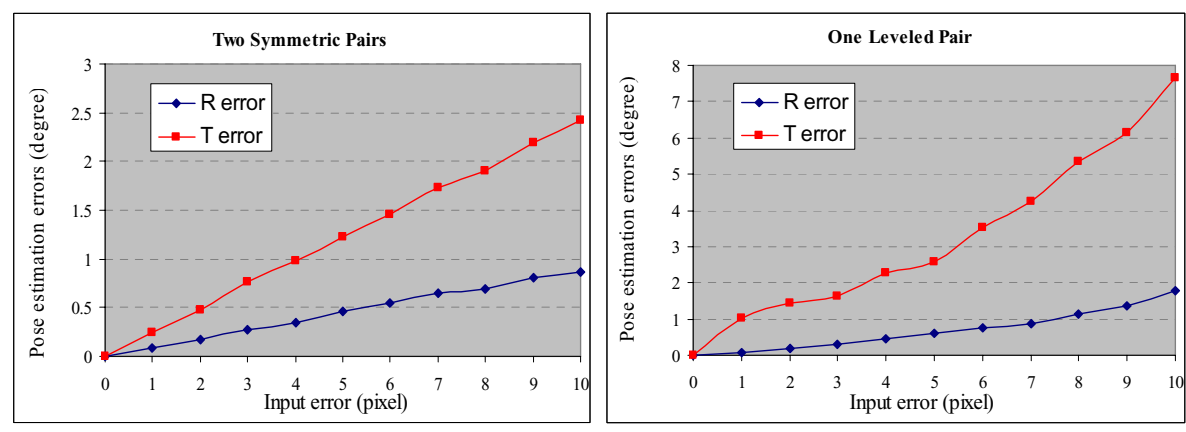

Fig. 8. Error sensitivity analysis for the symmetric or leveled case (synthetic images)

We used the SVD method for estimating $\hat{\mathbf{R}}$ and $\hat{\mathbf{T}}$ when symmetric pairs were used. We obtained $0.54^{\circ}$ estimation error for the rotation and $1.85^{\circ}$ estimation error for the translation. When only the leveled pair were used, due to the nonlinear constraints, the quadratic programming optimization approach was not directly applicable. Thus, the sequential quadratic programming method was used instead for optimization (i.e., function fmincon in MatLab). We obtained $1.22^{\circ}$ error in the rotation estimation and $4.65^{\circ}$ error in the translation. We show in Fig. 7 three particular epipolar curves calculated based on the erroneous estimations from the leveled case. The average $y$-difference between the identified corresponding points and the calculated epipolar curves is 1.2 pixel. For most points, those pose estimation errors, in this example, cause less than three pixel error in vertical direction while processing stereo matching.

We also conducted an error sensitivity analysis with simulated image data, in analogy to the real-world experiment, for both estimation approaches. Figure 8 plots how errors in detecting corresponding points impact the estimation result. The horizontal axes show various error sizes up to ten pixel. In the analysis, for example, a five-pixel input error means that each pair of corresponding image points was corrupted by errors of max/min five pixel in both $x$ - and $y$-values, and the errors are modeled as Gaussian-distributed random numbers.

In the case of symmetric panoramas, the curves (actually, nearly lines) of the estimation errors for rotation and translation show both a monotonic increase measured in the average for 500 runs. For up to ten-pixel input error, the estimation errors of rotation matrix or translation vector are less than one or three degrees, respectively. This analysis suggests that we had input errors of about six to eight pixel in our real-world experiment. However, this conclusion did not match our expectations. Hence, a further error analysis was conducted to test how the sensor calibration errors of $R$ and $\omega$ affect the pose estimation results. In the symmetric case, $R$ is an independent variable; and if $\omega$ has a one-degree error, then it would produce a four-pixel error in the input data. Therefore, the accuracy of sensor calibration, especially for $\omega$, is crucial to the pose estimation result.

For the case of leveled panoramas, the errors for both $\hat{\mathbf{R}}$ and $\hat{\mathbf{T}}$ are about two point five times the errors in the symmetric-panorama case. It suggests that 
the quadratic programming approach is more sensitive to input errors than the SVD method. Also, the assignment of initial values has significant impact onto the estimation result. According to our experiments, the estimation result was mostly sensitive to the 'sign' of the initial values but not to their quantities nor inter-ratios. In particular, zeros were not good for an initial guess in our case. The plots in this case indicate that we had input errors of about eight to nine pixel in our real-world experiment, which are close but slightly bigger than the conclusion drawn in the symmetric-panorama case. Error analysis on $R$ and $\omega$ was carried out as well. It concludes that the error of $R$ has a very minor impact on the pose estimation results. Moreover, a $k$-degree error of $\omega$ would cause about a $k$-degree error in the estimated $\hat{\mathbf{T}}$, for any real number $k$, but an error in $\omega$ has very little impact on the estimation of $\mathbf{R}$. The conclusion drawn here is coherent to the symmetric-panorama case.

Finally, more synthetic experiments were designed and performed for different panorama configurations (i.e., different poses, different sensor parameter values, and etc.). They lead to conclusions that the resolution of the input panoramic images, and the distribution of the selected corresponding points are also two critical factors for pose estimation. The panoramic image resolution, especially the width, should be as large as possible. The corresponding points should be distributed uniformly and sparsely on the entire panoramic images. A larger set of corresponding points, say greater than 100, would not guarantee a better estimation result. A much better result can be achieved if image resolution of $1,000 \times 10,000$ is used instead, and the nearest scene point is no less than four meters from both sensors. The estimation errors can be less than 0.5 degrees for both $\mathbf{R}$ and $\mathbf{T}$, allowing for both cases even up to ten-pixel input error.

\section{Conclusions}

This paper presented approaches for pose estimation of multi-view (i.e., also multi-center) cylindrical panoramas. Two geometric constraints were used: one was by coplanarity of corresponding projection rays, and the other was by intersection of corresponding projection rays.

The first constraint was used in the case of two symmetric pairs, and we obtained a "linear" solution for the sensor pose estimation problem. We showed that the (common) normalized 8-point algorithm can be utilized in this case. Experimentally, we found that the normalization step of the normalized 8-point algorithm for improving the accuracy and satiability was ignorable in our case 1 and this makes a difference to the planar image case. The second constraint was applied to cases of a general pair, or a leveled pair of cylindrical panoramas. Rather poor estimation results were obtained in the case of general pairs, and we did not include those here. In contrast to that, the result for the leveledpanorama case was greatly improved and reasonably stable. The proposed approaches are able to achieve high accuracy of less than 0.5 degree error in general,

${ }^{1}$ The corresponding image points on panoramas is likely not as skewed or clustered as in the planar image case. 
if high-resolution panoramic images are used and corresponding image points are carefully selected.

According to our error sensitivity analysis, the estimation of $\mathbf{T}$ is generally more sensitive to noise than the estimation of $\mathbf{R}$, and both estimation errors have approximately a linear relation to the input errors (as concluded from extensive simulations). We may also conclude that sensor pose estimation from leveled panoramas is more sensitive to errors than from pairs of symmetric panoramas. Moreover, the sensor calibration results of $R$ and $f$ have very little impact on pose estimation results, while $\omega$ 's error has a more serious influence on the accuracy of estimated sensors poses. For future work it is thus of interest to develop an algorithm, or a framework, that takes care of sensor calibration and pose estimation at once, similar to self-calibration for the planar image case.

\section{References}

1. Huang, F., Klette, R., Scheibe, K.: Panoramic Imaging: Sensor-Line Cameras and Laser Range-Finders. Wiley, West Sussex (2008)

2. Huang, F., Wei, S.K., Klette, R.: Geometrical fundamentals of polycentric panoramas. In: Proc. ICCV 2001, Vancouver, Canada, pp. I560-I565 (July 2001)

3. Li, Y., Shum, H.Y., Tang, C.K., Szeliski, R.: Stereo reconstruction from multiperspective panoramas. IEEE Transactions on Pattern Analysis and Machine Intelligence 26(1), 45-62 (2004)

4. Murray, D.: Recovering range using virtual multicamera stereo. CVIU 61(2), 285291 (1995)

5. Peleg, S., Ben-Ezra, M.: Stereo panorama with a single camera. In: Proc. CVPR 1999, Fort Collins, Colorado, USA, pp. 395-401 (June 1999)

6. Scheibe, K., Suppa, M., Hirschmäller, H., Strackenbrock, B., Huang, F., Liu, R., Hirzinger, G.: Multi-scale 3d-modeling. In: Chang, L.-W., Lie, W.-N. (eds.) PSIVT 2006. LNCS, vol. 4319, pp. 96-107. Springer, Heidelberg (2006)

7. Shum, H.Y., He, L.W.: Rendering with concentric mosaics. In: Proc. SIGGRAPH 1999, Los Angeles, California, USA, pp. 299-306 (August 1999)

8. Ishiguro, H., Yamamoto, M., Tsuji, S.: Omni-directional stereo. PAMI 14(2), $257-$ $262(1992)$

9. Kang, S.B., Szeliski, R.: 3-d scene data recovery using omnidirectional multibaseline stereo. IJCV 25(2), 167-183 (1997)

10. Seitz, S.: The space of all stereo images. In: Proc. ICCV 2001, Vancouver, Canada, pp. 26-33 (July 2001)

11. Hartley, R., Zisserman, A.: Multiple View Geometry in Computer Vision. Cambridge Uni. Press, United Kingdom (2000)

12. Chen, S.E.: QuickTimeVR - an image-based approach to virtual environment navigation. In: Proc. SIGGRAPH 1995, Los Angeles, California, USA, pp. 29-38 (August 1995)

13. Kang, S.B., Desikan, P.: Virtual navigation of complex scenes using clusters of cylindrical panoramic images. In: Graphics Interface, pp. 223-232 (1998) 